\title{
Accidental cloning of a single photon qubit in two-channel continuous-variable quantum teleportation
}

\author{
Toshiki Ide \\ Okayama Institute for Quantum Physics, \\ 1-9-1 Kyoyama, Okayama City, \\ Okayama, 700-0015, Japan \\ e-mail: toshiki_ide@pref.okayama.jp \\ Holger F. Hofmann \\ JST-CREST, Graduate School of Advanced Sciences of Matter, \\ Hiroshima University, \\ Kagamiyama 1-3-1, Higashi Hiroshima 739-8530, Japan \\ e-mail: hofmann@hiroshima-u.ac.jp
}

\begin{abstract}
The information encoded in the polarization of a single photon can be transferred to a remote location by two-channel continuous variable quantum teleportation. However, the finite entanglement used in the teleportation causes random changes in photon number. If more than one photon appears in the output, the continuous variable teleportation accidentally produces clones of the original input photon. In this paper, we derive the polarization statistics of the $N$-photon output components and show that they can be decomposed into an optimal cloning term and completely unpolarized noise. We find that the accidental cloning of the input photon is nearly optimal at experimentally feasible squeezing levels, indicating that the loss of polarization information is partially compensated by the availability of clones.
\end{abstract}

PACS numbers: 03.67.-a, 03.67.Hk, 42.50.-p, 03.65.Ud

\section{INTRODUCTION}

One of the most fascinating aspects of quantum optics is the insight it offers into the relation between the continuous field variables and photon numbers. In many cases, quantum protocols can be implemented by using either a photon or a continuous variable approach. In particular, this is true for teleportation and cloning, where both approaches have been realized experimentally [1, 2, 3, 4, 4, 5, 6]. Recently, there have also been efforts to combine both approaches, e.g. by applying homodyne detection to photon number states [7, 8], or by adding and subtracting photons from squeezed and coherent light $[9,10,11$. In the light of these technological advances, it is interesting to take a closer look at some of the possibilities inherent in the application of continuous-variable protocols to photon number states.

Since continuous-variable teleportation works for any input state, it is in principle straightforward to apply it to photon number inputs [12, 13, 14, 15]. However, the transmission process does not preserve photon number, so it is necessary to evaluate the effects of photon loss and photon addition. Specifically, a qubit encoded in the polarization state of a single photon can be either lost or multiplied in the continuous-variable teleportation process. If the photon is multiplied, the quantum information carried by its polarization is distributed to all output photons, resulting in an accidental cloning of the initial qubit. Photon multiplication errors should therefore be evaluated in terms of their cloning fidelity, which is of course limited by the fact that ideal cloning of quantum states is impossible [16, 17].

In the present paper, we analyze the photon number statistics in the output of a qubit teleportation and show that it can be decomposed into an optimal cloning term and completely unpolarized white noise. We derive the cloning fidelities and show that they are close to the optimal cloning fidelities at experimentally feasible squeezing levels. This result indicates that the transfer of quantum information is mostly limited by the availability of clones in the output. Interestingly, photons are cloned even though the transmitted field signal is not amplified. As our discussion shows, the cloning effects can be quantified in terms of the Gaussian field noise added in the teleportation process. We can therefore conjecture that accidental cloning is a general effect of Gaussian field noise on photonic qubits.

The rest of the paper is organized as follows. In section [1] we formulate the transfer operator for the continuousvariable teleportation of polarized light. In section ЏII we apply the formalism to a single photon state of unknown polarization and show that the output density matrix can be separated into a mixture of optimal clones and unpolarized white noise. In section IV] the $N$-photon outputs are identified and the cloning fidelities are derived. Finally, we summarize the results and their possible relevance in section $\nabla$. 


\section{TRANSFER OF POLARIZATION BY TWO-MODE CONTINUOUS-VARIABLE TELEPORTATION}

Conventional continuous-variable teleportation transfers only a single mode with a well defined polarization. In order to transfer the polarization of a single photon, it is therefore necessary to teleport two modes in parallel. Since continuous-variable teleportation preserves the coherence of the modes, it is not important which pair of orthogonal polarization modes is selected, as long as the four mode entangled state used in the teleportation is unpolarized $[15,18]$.

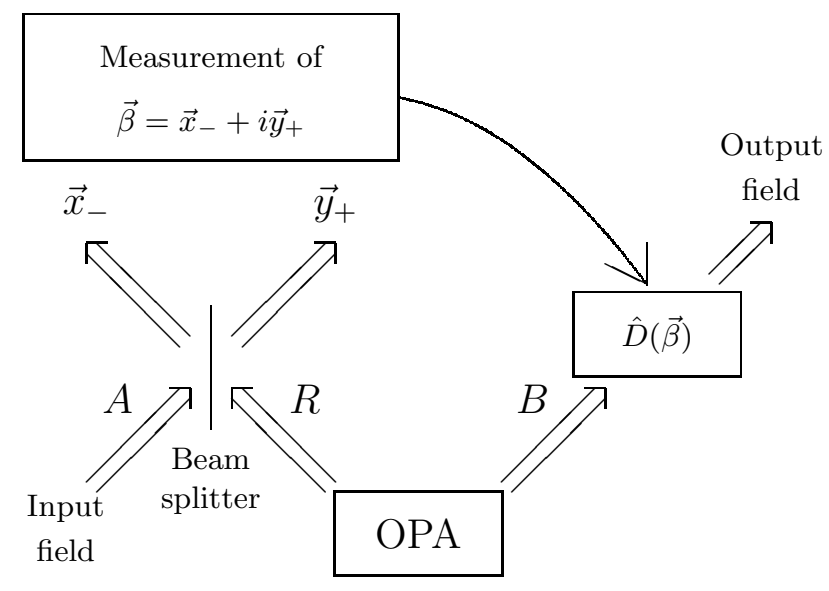

FIG. 1: Schematic representation of the two-mode quantum teleportation setup. The entangled state is generated by four-mode squeezing in an optical parametric amplifier (OPA). Four separate homodyne detection measurements are used to obtain the polarization components of the complex displacement amplitudes $\vec{\beta}=\left(\beta_{H}, \beta_{V}\right)$, and the corresponding complex two mode displacement amplitude is added to the output field $B$.

Fig. 11illustrates the extension of continuous-variable teleportation to the two mode system using the Jones vectors of the fields to express the polarization. The teleportation setup uses the four mode squeezed state generated by an optical parametric amplifier (OPA) to generate entanglement between two polarization modes in beam $R$ and two polarization modes in beam $B$. In the photon number basis of the horizontally $(H)$ and vertically $(V)$ polarized modes, this four-mode squeezed entangled state can be written as

$$
|\operatorname{EPR}(q)\rangle_{R, B}=\left(1-q^{2}\right) \sum_{N=0}^{\infty} q^{N} \sum_{n=0}^{N}|n ; N-n\rangle_{R}|n ; N-n\rangle_{B} .
$$

The amount of squeezing is given by $q(0 \leq q<1)$, which is related to the logarithmic attenuation of the field $r$ by $q=\tanh r$. Since the amount of squeezing is the same for all polarizations, the entangled state is completely unpolarized and the entanglement between the polarizations of $R$ and $B$ in each $N$-photon subspace is maximal.

The teleportation is then performed by mixing the two mode input in beam $A$ with the reference $R$ at a $50 / 50$ beam splitter and measuring the quadrature components $\vec{x}_{-}$of the difference and $\vec{y}_{+}$of the sum. In practice, this requires four separate homodyne detection measurements, to obtain the real and imaginary parts of the two dimensional Jones vector, $\vec{\beta}=\vec{x}_{-}+i \vec{y}_{+}$. However, the choice of polarizations for the Jones vector measurement has no effect on the teleportation itself since the Bell measurement performed on $R$ and $A$ simply projects the two beams onto a maximally entangled state displaced by a coherent amplitude of $\vec{\beta}$ [19]. Due to the entanglement between $R$ and $B$, the field value $\vec{\beta}$ corresponds to a field difference between the unknown input $A$ and the output beam $B$. This difference can be corrected by a two mode field displacement $\hat{D}_{\text {pol }}(\vec{\beta})$. The complete teleportation process can then be summarized by a two mode transfer operator, which is a straightforward extension of the single-mode case derived in [19]. This operator reads

$$
\hat{T}_{\mathrm{pol}}(\vec{\beta})=\frac{1-q^{2}}{\pi} \sum_{N=0}^{\infty} \sum_{n=0}^{N} q^{N} \hat{D}_{\mathrm{pol}}(\vec{\beta}) \hat{\Pi}_{N} \hat{D}_{\mathrm{pol}}(-\vec{\beta})
$$


where the operator $\hat{\Pi}_{N}$ is the projection operator of the $N$ photon subspace,

$$
\hat{\Pi}_{N}=\sum_{n=0}^{N}|n, N-n\rangle\langle n, N-n| .
$$

It should be noted that the choice of polarization modes in the preparation of the squeezed state and in the homodyne measurements have no effect on the transfer process itself. The transfer operator is completely defined by the Jones vector $\vec{\beta}$ obtained in the homodyne detections.

As explained in [19], the transfer operator determines both the probabilities of measuring $\vec{\beta}$ and the output state of the teleportation. The output density matrix is a mixture over all possible measurement outcomes given by

$$
\hat{\rho}_{\text {out }}=\int d^{4} \vec{\beta} \hat{T}_{\text {pol }}(\vec{\beta})\left|\psi_{\text {in }}\right\rangle\left\langle\psi_{\text {in }}\right| \hat{T}_{\text {pol }}^{\dagger}(\vec{\beta}) .
$$

In this integral, the Jones vector $\vec{\beta}$ is averaged over all possible polarizations, so the polarization of the output depends only on the input polarization.

Since it will be relevant in the following analysis, it may be useful to consider the case of a vacuum input. In this case, the teleportation simply adds Gaussian noise to the field, resulting in a thermal state output given by

$$
\begin{aligned}
\hat{R}_{\mathrm{vac}} & =\int d^{4} \vec{\beta} \hat{T}_{\mathrm{pol}}(\vec{\beta})|0 ; 0\rangle\langle 0 ; 0| \hat{T}_{\mathrm{pol}}^{\dagger}(\vec{\beta}) \\
& =\left(\frac{1+q}{2}\right)^{2} \sum_{N}\left(\frac{1-q}{2}\right)^{N} \hat{\Pi}_{N} .
\end{aligned}
$$

In the wave picture, the teleportation error can be interpreted as Gaussian field noise with a variance of $V_{q}$ equal to the average photon number added to each mode [20]. According to the thermal state given by (5), this error is related to the squeezing parameter $q$ by

$$
V_{q}=\frac{1-q}{1+q}
$$

The value of the teleportation error $V_{q}$ lies between zero for error free teleportation with infinitely squeezed light, and one for the limit of classical teleportation using a pair of vacuum states instead of entanglement. In the particle picture, $V_{q}$ is the average number of photons added per mode, so two-mode teleportation adds a total average of $2 V_{q}$ photons in the output field.

Since the vacuum state input is completely unpolarized, the output state has no polarization either. However, the teleportation process will transfer the polarization of the input state to the output. In the next section, we will show how the transfer operator can be used to describe this transfer of polarization in the case of a single-photon polarization qubit.

\section{CONTINUOUS-VARIABLE TELEPORTATION OF A SINGLE-PHOTON POLARIZATION QUBIT}

We now consider the case of a single photon input of unknown polarization. Such input states can be described by a superposition of two basis states, $|H\rangle=\hat{a}_{H}^{\dagger}|0 ; 0\rangle$ and $|V\rangle=\hat{a}_{V}^{\dagger}|0 ; 0\rangle$. Alternatively, the unknown quantum information can be described by a creation operator $\hat{a}_{\text {in }}^{\dagger}$, so that the input state is given by

$$
\begin{aligned}
\left|\psi_{\text {in }}\right\rangle & =\hat{a}_{\text {in }}^{\dagger}|0 ; 0\rangle \\
\text { where } \quad \hat{a}_{\text {in }} & =c_{H}^{*} \hat{a}_{H}+c_{V}^{*} \hat{a}_{V} .
\end{aligned}
$$

By using the basis independent properties of the operator $\hat{a}_{\text {in }}$, we can keep track of the quantum information in the single photon input as it is transferred to the multi photon output components.

According to the transfer operator formalism, the output density matrix for the single photon qubit teleportation is given by

$$
\hat{\rho}_{\text {out }}=\int d^{4} \vec{\beta} \hat{T}_{\text {pol }}(\vec{\beta}) \hat{a}_{\text {in }}^{\dagger}|0 ; 0\rangle\langle 0 ; 0| \hat{a}_{\text {in }} \hat{T}_{\text {pol }}^{\dagger}(\vec{\beta})
$$


To solve this integral, we need to consider the effects of the transfer operator on the unknown operator $\hat{a}_{\text {in }}$, which defines the polarization of the input qubit. For this purpose, it is convenient to define the component $\beta_{\text {in }}$ of the Jones vector $\vec{\beta}$ with the same polarization as the unknown input,

$$
\beta_{\mathrm{in}}=c_{H}^{*} \beta_{H}+c_{V}^{*} \beta_{V}
$$

It is then possible to commute the transfer operators $\hat{T}_{\text {pol }}(\vec{\beta})$ and the operators of the unknown input polarization, $\hat{a}_{\text {in }}$ and $\hat{a}_{\text {in }}^{\dagger}$, using the relations

$$
\begin{aligned}
& \hat{T}_{\mathrm{pol}}(\vec{\beta}) \hat{a}_{\text {in }}^{\dagger}=\left(q \hat{a}_{\text {in }}^{\dagger}+(1-q) \beta_{\text {in }}^{*}\right) \hat{T}_{\text {pol }}(\vec{\beta}) \\
& \hat{a}_{\text {in }} \hat{T}_{\text {pol }}(\vec{\beta})=\hat{T}_{\text {pol }}(\vec{\beta})\left(q \hat{a}_{\text {in }}+(1-q) \beta_{\text {in }}\right) .
\end{aligned}
$$

The output density matrix can then be written as

$$
\hat{\rho}_{\text {out }}=\int d^{4} \vec{\beta}\left(q^{2} \hat{a}_{\text {in }}^{\dagger} \hat{r}_{\mathrm{vac}} \hat{a}_{\text {in }}+(1-q) q\left(\hat{a}_{\text {in }}^{\dagger} \beta_{\text {in }} \hat{r}_{\mathrm{vac}}+\hat{r}_{\mathrm{vac}} \beta_{\text {in }}^{*} \hat{a}_{\text {in }}\right)+(1-q)^{2} \beta_{\text {in }} \hat{r}_{\mathrm{vac}} \beta_{\text {in }}^{*}\right),
$$

where $\hat{r}_{\text {vac }}$ is an abbreviation for the operator obtained by applying the transfer operator to the vacuum density matrix,

$$
\hat{r}_{\mathrm{vac}}(\vec{\beta})=\hat{T}_{\mathrm{pol}}(\vec{\beta})|0 ; 0\rangle\langle 0 ; 0| \hat{T}_{\mathrm{pol}}^{\dagger}(\vec{\beta})
$$

Since $\beta_{\text {in }}$ is unknown, the integral in eq.111 still depends on the coefficients $c_{H}$ and $c_{V}$ defining the direction of the unknown input polarization. To obtain an integral that is independent of the unknown polarization, we need to convert these values back into operators independent of the measurement outcome $\vec{\beta}$. This transformation can be accomplished by making use of the fact that the vacuum teleportation $\hat{T}_{\text {pol }}(\vec{\beta})|0 ; 0\rangle$ results in a coherent state with an amplitude of $(1-q) \vec{\beta}[19]$. Therefore, this state is a right eigenstate of $\hat{a}_{\text {in }}$, and we can transform $\beta_{\text {in }}$ into $\hat{a}_{\text {in }}$ using

$$
\begin{aligned}
& \hat{a}_{\text {in }} \hat{r}_{\mathrm{vac}}=(1-q) \beta_{\text {in }} \hat{r}_{\mathrm{vac}} \\
& \hat{r}_{\mathrm{vac}} \hat{a}_{\text {in }}^{\dagger}=(1-q) \beta_{\text {in }}^{*} \hat{r}_{\mathrm{vac}} .
\end{aligned}
$$

It is thus possible to convert all factors of $\beta_{\text {in }}$ in eq. (11), resulting in an integral where only $\hat{r}_{\text {vac }}$ depends on $\vec{\beta}$,

$$
\hat{\rho}_{\text {out }}=\int d^{4} \vec{\beta}\left(q^{2} \hat{a}_{\text {in }}^{\dagger} \hat{r}_{\mathrm{vac}} \hat{a}_{\text {in }}+q\left(\hat{a}_{\text {in }}^{\dagger} \hat{a}_{\text {in }} \hat{r}_{\mathrm{vac}}+\hat{r}_{\mathrm{vac}} \hat{a}_{\text {in }}^{\dagger} \hat{a}_{\text {in }}\right)+\hat{a}_{\text {in }} \hat{r}_{\mathrm{vac}} \hat{a}_{\text {in }}^{\dagger}\right) .
$$

We can now perform the integration of $\hat{r}_{\mathrm{vac}}$, the result of which is equal to the output state of vacuum teleportation

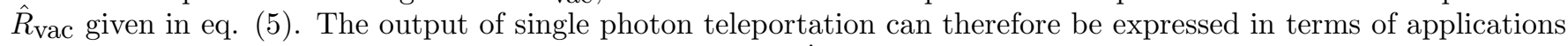
of the input operator $\hat{a}_{\text {in }}$ to the unpolarized thermal state $\hat{R}_{\text {vac }}$,

$$
\hat{\rho}_{\text {out }}=q^{2} \hat{a}_{\text {in }}^{\dagger} \hat{R}_{\mathrm{vac}} \hat{a}_{\text {in }}+q\left(\hat{R}_{\mathrm{vac}} \hat{a}_{\text {in }}^{\dagger} \hat{a}_{\text {in }}+\hat{a}_{\text {in }}^{\dagger} \hat{a}_{\text {in }} \hat{R}_{\mathrm{vac}}\right)+\hat{a}_{\text {in }} \hat{R}_{\mathrm{vac}} \hat{a}_{\text {in }}^{\dagger}
$$

The result can be simplified by considering the commutation relations of the thermal state and arbitrary creation and annihilation operators, namely

$$
\begin{aligned}
& \hat{a}_{\mathrm{in}} \hat{R}_{\mathrm{vac}}=\frac{1-q}{2} \hat{R}_{\mathrm{vac}} \hat{a}_{\mathrm{in}} \\
& \hat{R}_{\mathrm{vac}} \hat{a}_{\text {in }}^{\dagger}=\frac{1-q}{2} \hat{a}_{\text {in }}^{\dagger} \hat{R}_{\mathrm{vac}} .
\end{aligned}
$$

It is then possible to rearrange the operator ordering in eq.(15) so that the operator $\hat{a}_{\text {in }}$ acts only as a creation operator on the thermal output $\hat{R}_{\mathrm{vac}}$. In this simplified form, the output reads

$$
\hat{\rho}_{\text {out }}=\left(\frac{1+q}{2}\right)^{2} \underbrace{\hat{a}_{\text {in }}^{\dagger} \hat{R}_{\mathrm{vac}} \hat{a}_{\text {in }}}_{\text {photon added state }}+\left(\frac{1-q}{2}\right) \underbrace{\hat{R}_{\text {vac. }}}_{\text {white noise }}
$$


As indicated, it is now possible to interpret the output as a mixture of a photon added state polarized by the application of the creation operator $\hat{a}_{\text {in }}^{\dagger}$ to $\hat{R}_{\mathrm{vac}}$, and a completely unpolarized white noise component represented by the thermal state $\hat{R}_{\mathrm{vac}}$ itself. It may be interesting to note that the photon added state is a two mode version of the single photon added thermal state investigated in recent experiments [21, 22] because of its non-classical features such as the negativity of the Wigner function [23]. The photon added term in eq.(17) thus describes the teleportation of non-classical features in the field quadrature statistics of the single photon state. For the purpose of photon cloning however, only the photon number distributions are relevant. In that context, it is significant that the application of a creation operator also describes the effects of photon bunching and of stimulated emission used in previous photon cloning experiments [3, 4, 5]. As we shall show in the following, the photon added state is indeed equivalent to a mixture of optimally cloned $N$-photon outputs. It is therefore possible to interpret the photon added state as optimal cloning and the thermal white noise background as a measure of the non-optimal nature of accidental cloning.

More specifically, eq.(17) provides a simple quantification of the unpolarized white noise background added in the teleportation in terms of the statistical weights of the two components. Since $\hat{R}_{\mathrm{vac}}$ is normalized, the statistical weights are $(1-q) / 2$ for the white noise and $(1+q) / 2$ for the photon added state representing optimal cloning. The ratio of the two components is thus exactly equal to the Gaussian field error $V_{q}$ derived in sec. II. Since $V_{q}$ is a very intuitive measure of the teleportation error in terms of field noise, it may be interesting to see how this continuous variable noise measure is related to the cloning errors in the discrete photon number statistics.

\section{CLONING FIDELITY OF THE $N$-PHOTON OUTPUT}

In the previous section, we have derived the complete output state of the two mode teleportation of a single photon polarization qubit. The total output photon number of this state is random, so it is not possible to predict how many clones (if any) are generated. Since the optimal cloning fidelity is a function of the number of clones produced, it is necessary to separate the output density matrix of eq.(17) into its $N$-photon output components, representing the accidental occurrences of $1 \rightarrow N$ cloning events,

$$
\hat{\rho}_{\text {out }}=\sum_{N=0}^{\infty} P(N) \hat{\rho}_{N} .
$$

The decomposition of the unpolarized thermal states $\hat{R}_{\mathrm{vac}}$ in eq.(17) is given by eq.(5). In the photon added component that represents optimal cloning, the number of photons is raised by one due to the application of the creation operator $\hat{a}_{\text {in }}^{\dagger}$. Therefore, the decomposition of the output density matrix into $N$-photon subspaces includes a white noise term given by $\hat{\Pi}_{N}$ and an optimal cloning term given by $\hat{a}_{\text {in }}^{\dagger} \hat{\Pi}_{N-1} \hat{a}_{\text {in. }}$. Since the optimal cloning part has no zero-photon component, $\hat{\Pi}_{-1}$ should be defined as zero. The decomposition of the output density matrix into $N$-photon subspaces then reads

$$
\begin{aligned}
\hat{\rho}_{\text {out }} & =\frac{(1+q)^{2}}{2(1-q)} \sum_{N=0}^{\infty}\left(\frac{1-q}{2}\right)^{N}\left(\left(\frac{1+q}{2}\right)^{2} \hat{a}_{\text {in }}^{\dagger} \hat{\Pi}_{N-1} \hat{a}_{\text {in }}+\left(\frac{1-q}{2}\right)^{2} \hat{\Pi}_{N}\right) \\
& =\frac{1}{V_{q}\left(1+V_{q}\right)^{3}} \sum_{N=0}^{\infty}\left(\frac{V_{q}}{1+V_{q}}\right)^{N}\left(\hat{a}_{\text {in }}^{\dagger} \hat{\Pi}_{N-1} \hat{a}_{\text {in }}+V_{q}^{2} \hat{\Pi}_{N}\right)
\end{aligned}
$$

Here, the entanglement parameter $q$ has been converted into the more intuitive measure of Gaussian field error $V_{q}$. It is then possible to relate the photon number distribution and the cloning errors directly to the Gaussian noise error of the continuous variable teleportation.

The statistical weights of the contributions in eq.(19) are determined by the traces of the operators. Specifically, the trace of the white noise term $\hat{\Pi}_{N}$ is $N+1$ and the trace of the optimal cloning term $\hat{a}_{\text {in }}^{\dagger} \hat{\Pi}_{N-1} \hat{a}_{\text {in }}$ is $(N+1) N / 2$. The probability $P(N)$ of obtaining an $N$-photon output is given by the product trace of the total density matrix in the $N$-photon subspace. In terms of the Gaussian field noise $V_{q}$, this photon number distribution reads

$$
\begin{aligned}
P(N) & =\operatorname{Tr}\left\{\hat{\Pi}_{N} \hat{\rho}_{\text {out }}\right\} \\
& =\frac{(N+1)\left(N+2 V_{q}^{2}\right)}{2 V_{q}\left(1+V_{q}\right)^{3}}\left(\frac{V_{q}}{1+V_{q}}\right)^{N} .
\end{aligned}
$$

$P(0)$ is the probability of losing the photon, $P(1)$ is the probability of single photon teleportation, and $P(N \geq 2)$ are the probabilities of accidental $1 \rightarrow N$ cloning. Since the generation of additional photons is itself a kind of 
teleportation error, the probability of generating accidental clones increases with $V_{q}$. Specifically, the average photon number in the output is $1+2 V_{q}$ (the original photon plus twice the average photon number added per mode). Even at $V_{q}=1$, the average photon number is only three and the probability of obtaining a high number of output photons drops with $(1 / 2)^{N}$. Accidental cloning probabilities are therefore generally low for high numbers of clones. However, the probabilities of obtaining two, three or four clones can be quite significant, as shown in fig.2. Specifically, the probabilities of obtaining $N$ clones at an experimentally feasible error of $V_{q}=0.25$ requiring about $6 \mathrm{~dB}$ squeezing are $P(2)=26.1 \%, P(3)=10.2 \%$, and $P(4)=3.4 \%$. The accidental generation of clones should therefore be a very common occurrence if qubits are teleported using squeezed state entanglement at presently available levels of squeezing.

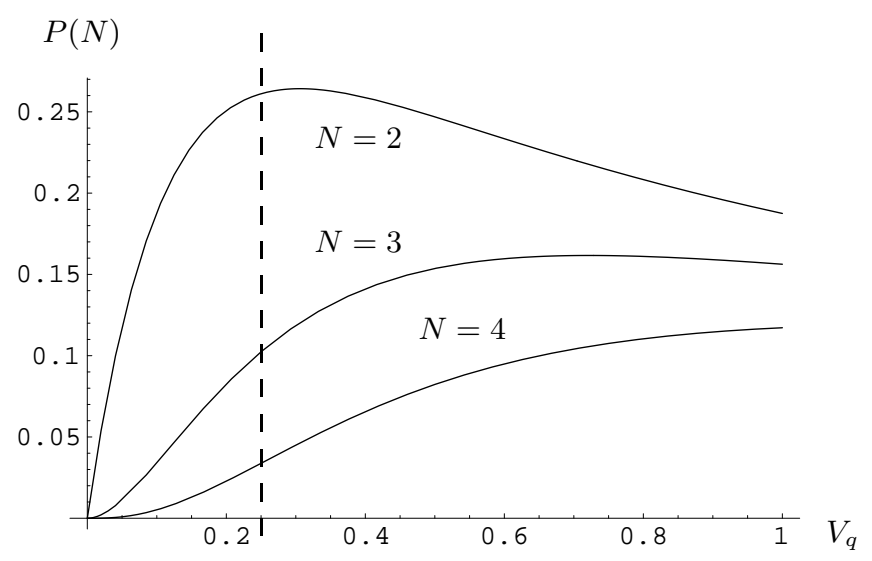

FIG. 2: Cloning probabilities $P(N)$ for different output photon numbers $N$ plotted to $V_{q}$. From top to bottom, the curves show $P(2), P(3), P(4)$. The dashed vertical line indicates $V_{q}=0.25$.

We can now evaluate the quality of accidental $1 \rightarrow N$ cloning by separating the $N$-photon output $\hat{\rho}_{N}$ into its optimal cloning component $\hat{C}_{N}$ and its white noise component $\hat{W}_{N}$,

$$
\hat{\rho}_{N}=\eta_{N} \hat{C}_{N}+\left(1-\eta_{N}\right) \hat{W}_{N} .
$$

The density matrices $\hat{C}_{N}$ and $\hat{W}_{N}$ are independent of the teleportation errors $V_{q}$ and can be generated from the projection operators $\hat{\Pi}_{N}$ into the $N$-photon subspaces by

$$
\begin{aligned}
\hat{C}_{N} & =\frac{2}{N(N+1)} \hat{a}_{\text {in }}^{\dagger} \hat{\Pi}_{N-1} \hat{a}_{\text {in }} \\
\hat{W}_{N} & =\frac{1}{N+1} \hat{\Pi}_{N} .
\end{aligned}
$$

Therefore, the $N$-photon output is fully characterized by the single parameter $\eta_{N}$. Since this parameter defines the fraction of optimally cloned $N$-photon outputs, we will call it the cloning efficiency of accidental $1 \rightarrow N$ cloning. The cloning efficiency $\eta_{N}$ is a function of teleportation error $V_{q}$ and photon number $N$. Using eq.(17), we find that this ratio is

$$
\eta_{N}=\frac{1}{1+2 V_{q}^{2} / N}
$$

Fig. 3 illustrates this dependence of cloning efficiency $\eta_{N}$ on the teleportation error $V_{q}$ for several output photon numbers $N$. Not surprisingly, the teleportation error $V_{q}$ reduces the cloning efficiency. However, it is interesting to note that only the square of $V_{q}$ enters into the relation, indicating that the cloning efficiency rapidly approaches one for low teleportation errors. For example, an experimentally feasible error of $V_{q}=0.25$ requiring about $6 \mathrm{~dB}$ of squeezing already gives a two photon cloning efficiency of $\eta_{2}=16 / 17$, or about $94 \%$. We can therefore conclude that the accidental cloning observed in continuous variable teleportation at presently available levels of squeezed state entanglement will be nearly optimal. Another significant feature of accidental cloning is that the minimal cloning efficiencies obtained at the classical teleportation limit $\left(V_{q}=1\right)$ have a photon number dependent value of $N /(N+2)$. Thus, the cloning efficiencies for high $N$ are always close to one, indicating that the generation of a large number of clones is quite robust against teleportation errors. 


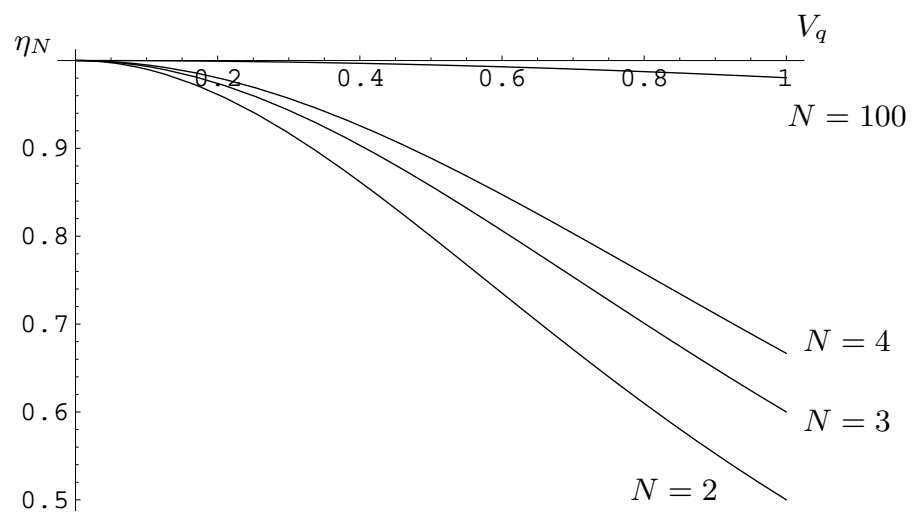

FIG. 3: Cloning efficiency $\eta_{N}$ for different output photon numbers $N$ plotted to $V_{q}$. From bottom to top, the curves show $\eta_{2}, \eta_{3}, \eta_{4}$ and $\eta_{100}$

Up to now, our discussion was based only on the formal decomposition of the output into an optimal cloning component and a white noise term. Experimentally, this decomposition is not directly observable. Instead, cloning is characterized by the cloning fidelity $F_{N}$, defined as the fraction of output photons with the same polarization as the input photon, $\left\langle\hat{a}_{\text {in }}^{\dagger} \hat{a}_{\text {in }}\right\rangle / N$. This fidelity is usually measured by splitting up the output light into a sufficiently large number of channels, so that the polarization of each photon can be detected separately [3, 5]. It is then possible to evaluate cloning by conventional photon counting.

To determine the fidelity $F_{N}$ of a $1 \rightarrow N$ cloning process with cloning efficiency $\eta_{N}$, we can use the fidelities of the optimal cloning component $\hat{C}_{N}$ and the white noise components $\hat{W}_{N}$. Since the white noise component is completely unpolarized, exactly half of the photons will have the same polarization as the input, corresponding to a fidelity of $1 / 2$. For the optimal cloning term, the cloning fidelity is given by

$$
F_{\text {opt. }}=\frac{1}{N} \operatorname{Tr}\left\{\hat{a}_{\text {in }}^{\dagger} \hat{a}_{\text {in }} \hat{C}_{N}\right\}=\frac{2 N+1}{3 N} .
$$

This result is equal to the $1 \rightarrow N$ cloning fidelity of an optimal cloning machine [17], proving our conjecture that $\hat{C}_{N}$ represents optimal cloning. The accidental cloning fidelity $F_{N}$ of the mixture of $\hat{C}_{N}$ and $\hat{W}_{N}$ defined by the cloning efficiency $\eta_{N}$ can now be obtained by taking the weighted average of the white noise fidelity of $1 / 2$ and the optimal cloning fidelity given by eq.(24),

$$
\begin{aligned}
F_{N} & =\eta_{N} \frac{2 N+1}{3 N}+\left(1-\eta_{N}\right) \frac{1}{2} \\
& =\frac{2}{3}+\frac{1-V_{q}^{2}}{3\left(N+2 V_{q}^{2}\right)} .
\end{aligned}
$$

As this relation shows, the cloning efficiencies at $V_{q}=1$ all correspond to a cloning fidelity of $2 / 3$, which is the optimal cloning fidelity for $N \rightarrow \infty$. Moreover, $2 / 3$ is also the optimal cloning fidelity obtained if the cloning is performed using only local measurements and classical communications instead of a direct quantum mechanical interaction between the original and the clones. At $V_{q}=1$, this is exactly what happens, since there is no entanglement and the teleportation is performed by a local measurement of the input photon and the generation of a coherent state of the appropriate amplitude in the output. It is therefore obvious that $V_{q}=1$ is still close to the optimal limit for high $N$. On the other hand, cloning fidelities above $2 / 3$ are only possible because of the entanglement used in the teleportation.

Fig 4 shows the cloning fidelity $F_{N}$ for two, three, and five output photons. In addition, the high $N$ limit is indicated by the result for $N=100$, which is basically indistinguishable from a flat line at $F_{\infty}=2 / 3$. The dotted line indicates a realistic teleportation error of $V_{q}=0.25$, achievable by about $6 \mathrm{~dB}$ of squeezing. The cloning fidelities at this noise level are already quite close to the optimal fidelities. Specifically, the fidelities at $V_{q}=0.25\left(V_{q}=0\right)$ are $F_{2}=0.814$ $\left(F_{2}=0.833\right), F_{3}=0.767\left(F_{3}=0.778\right), F_{4}=0.742\left(F_{4}=0.75\right)$. It should therefore be possible to observe nearly optimal accidental quantum cloning in the continuous variable teleportation of single photon qubits under presently realizable experimental conditions.

In order to put the above results into a wider context, it may be interesting to recall that the cloning effect has been obtained without any field amplification. In fact, a minimal noise amplification can produce optimal clones, and 


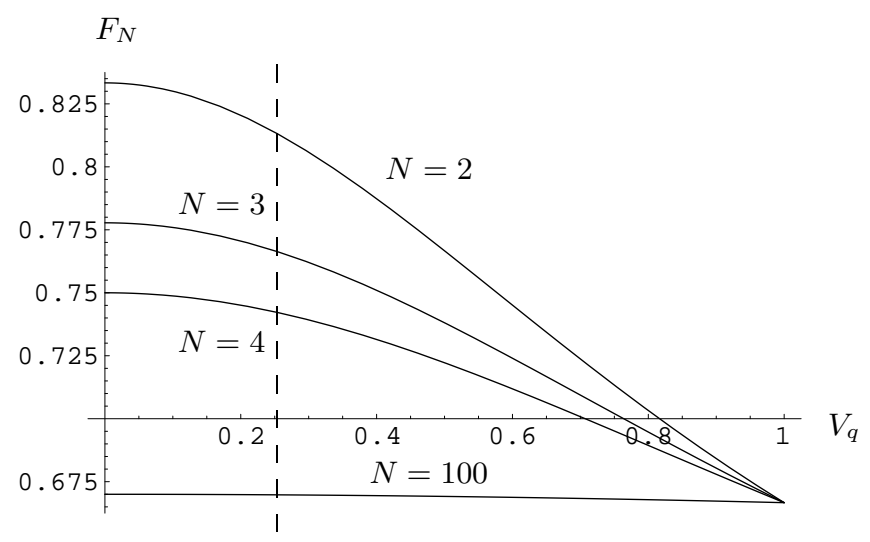

FIG. 4: Cloning fidelities $F_{N}$ for different output photon numbers $N$ plotted to $V_{q}$. From top to bottom, the curves show $F_{2}, F_{3}, F_{4}$ and $F_{100}$. The dashed vertical line indicates $V_{q}=0.25$.

this situation can be realized by adjusting the gain of continuous variable teleportation, as we have already pointed out elsewhere [24]. However, accidental cloning occurs at a gain of one, due to the Gaussian field noise added in the teleportation. Since all linear optics processes should be equivalent, we can conjecture that our results describe the accidental cloning effects of any kind of Gaussian field noise added to the field of a single photon qubit state. In particular, $V_{q}$ can be modified to include a variety of Gaussian errors in addition to the limitation of squeezed state entanglement. In this case, $V_{q}$ could even exceed one, indicating fidelities below $2 / 3$. However, all successful continuous variable teleportation experiments should reduce $V_{q}$ well below one, corresponding to cloning fidelities close to optimal cloning.

\section{CONCLUSIONS}

Our results show that the accidental multiplication of a single photon polarization qubit transferred by continuous variable teleportation can be interpreted as a nearly optimal quantum cloning process. A continuous-variable teleportation system operating at feasible squeezing levels thus tends to act both as a fax and a copy machine on the teleported qubits. Even though the cloning happens as a consequence of the noise introduced by non-maximal entanglement, the effect is similar to intentional telecloning, where a special multi-photon entangled state needs to be prepared beforehand [25]. Our analysis indicates that telecloning is a natural feature of the continuous-variable teleportation process when it is applied to single-photon qubits. This observation might be useful in the implementation of multi party protocols, where the distribution of quantum information to several parties is desirable.

It is also interesting to note that the clones are generated without field amplification, simply by the random addition of Gaussian field noise. We can therefore conjecture that accidental cloning is a general effect of Gaussian field noise on photonic qubits. Even though this kind of cloning without amplification can never be optimal, the additional error can be described by mixing the optimal cloning output with a completely unpolarized component of the density matrix. We have thus successfully converted a Gaussian field error into an $N$-photon cloning error, with the cloning efficiency $\eta_{N}$ describing the exact fraction of the optimal cloning state in the output. In the context of recent investigations into the quantum mechanics of the photon-field dualism [7, 8, 9, 10, 11], this result may shed some light on the fundamental relations between photons and field noise.

In conclusion, the accidental cloning of photonic qubits in continuous variable teleportation is a phenomenon that may be both useful in the development of new technologies for quantum information networks and in the exploration of the fundamental physics behind the dualism of photons and fields. Hopefully, the analysis presented above will be a fruitful contribution to both. 


\section{Acknowledgment}

Part of this work has been supported by the Grant-in-Aid program of the Japanese Society for the Advancement of Science and by the JST-CREST project on quantum information processing.

[1] D. Bouwmeester, J. W. Pan, K. Mattle, M. Eibl, H. Weinfurter and A. Zeilinger, Nature 390, 575 (1997).

[2] A. Furusawa, J. L. Sørensen, S. L. Braunstein, C. A. Fuchs, H. J. Kimble, and E. J. Polzik, Science 282, 706 (1998).

[3] A. Lamas-Linares, C. Simon, J. C. Howell and D. Bouwmeester, Science 296, 712 (2002).

[4] F. De Martini, V. Buz̃ek, F. Bovino and C. Sias, Nature 419, 815 (2002).

[5] W.T.M. Irvine, A.L. Linares, M.J.A. de Dood, and D. Bouwmeester, Phys. Rev. Lett. 92, 047902 (2004).

[6] U. L. Andersen, V. Josse and G. Leuchs Phys. Rev. Lett. 94, 240503 (2005).

[7] A. I. Lvovsky, H. Hansen, T. Aichele, O. Benson, J. Mlynek and S. Schiller, Phys. Rev. Lett. 87, 050402 (2001).

[8] A. I. Lvovsky and J. Mlynek, Phys. Rev. Lett. 88, 250401 (2002).

[9] T. Opatrny, G. Kurizki, D.-G. Welsch, Phys. Rev. A61 032302 (2000).

[10] A. Zavatta, S. Viciani, and M. Bellini, Science 306, 660 (2004).

[11] A. Ourjoumtsev, R. Tualle-Brouri, P. Grangier, Phys. Rev. Lett. 96, 213601 (2006).

[12] R. E. S. Polkinghorne and T. C. Ralph, Phys. Rev. Lett. 83, 2095 (1999).

[13] T.C. Ralph, Phys. Rev. A 61, 044301 (2001).

[14] T.C. Ralph, Phys. Rev. A 65, 012319 (2002).

[15] T. Ide, H. F. Hofmann, T. Kobayashi and A. Furusawa, Phys. Rev. A 65, 012313 (2002).

[16] W. K. Wooters and W. H. Zurek, Nature 299, 802 (1996).

[17] N. Gisin and S. Massar, Phys. Rev. Lett. 79, 2153 (1997).

[18] A. Dolinska, B.C. Buchler, W.P. Bowen, T.C. Ralph, and P.K. Lam, Phys. Rev. A 68, 052308 (2003).

[19] H. F. Hofmann, T. Ide, T. Kobayashi, and A. Furusawa, Phys. Rev. A, 62, 062304 (2000).

[20] S. L. Braunstein and H. J. Kimble, Phys. Rev. Lett. 80, 869 (1998).

[21] V. Parigi, A. Zavatta and M. Bellini, Proc. of SPIE 6305, 63050Z (2006).

[22] A. Zavatta, V. Parigi, and M. Bellini, e-print arXiv:0704.0179 (2007).

[23] G.S. Agarwal and K. Tara, Phys. Rev. A 46, 458 (1992).

[24] H. F. Hofmann and T. Ide, New J. Phys. 8, 130 (2006).

[25] M. Murao, D. Jonathan, M. B. Plenio and V. Vedral, Phys. Rev. A 59, 156 (1999). 